\title{
Green solid films with tunable mechanical properties made from deoxyribonucleic acid
}

\author{
Jie Zhan ${ }^{1}$, Hisao Matsuno ${ }^{1}$, Hiroyasu Masunaga ${ }^{2}$, Hiroki Ogawa ${ }^{2}$ and Keiji Tanaka ${ }^{1,3}$ \\ Promoting green innovation to establish a worldwide low-carbon society is an urgent priority. Effective utilization of natural \\ resources excluding fossil fuels is one of the efforts that will contribute to the resolution of this issue. Here, we show that \\ solid-state films made from deoxyribonucleic acid (DNA) can be used as a structural material. The great advantage of the DNA \\ films over the ones made from synthetic polymers is that the mechanical properties are controllable, from glassy to rubbery, \\ via semicrystalline by simply regulating the water content in the film. Why and how such unique mechanical properties can be \\ manifested by the DNA films is determined from detail structural analyses using Fourier-transform infrared spectroscopy and \\ wide-angle X-ray diffraction measurements. With an increasing water content, the conformation of double-stranded DNA \\ (dsDNA) was changed from the A-form in an amorphous state to the B-form in a partially packed one. dsDNA in the B-form \\ became densely packed as the film was stretched.
}

NPG Asia Materials (2014) 6, e92; doi:10.1038/am.2014.4; published online 14 March 2014

Keywords: DNA; green material; tunable mechanical properties; water

\section{INTRODUCTION}

In the past, many kinds of polymers which are of better quality than natural polymers have been chemically synthesized. Even now, the development of novel reactions and catalysts for polymerization are underway to improve the characteristics of synthetic polymeric materials. On the other hand, recently, the usage of natural polymers has attracted increasing attention for another reason-because of the consumption and overexploitation of nonrenewable resources such as oil and coal. Some natural polymers, such as natural rubber and cellulose, exhibit excellent mechanical properties that no synthetic polymer has yet achieved. Deoxyribonucleic acid (DNA) is also an attractive natural green polymer. ${ }^{1}$ DNA has been used not only as a genetic material for diagnosis and therapeutics but also as an engineering material for electrically conducting devices and sensors due to its abundant $\pi$-electrons contained in the base-pair portions. ${ }^{2-4}$

Despite the growth of the literature documenting the application of DNA as a solid material, the industrial use of DNA has made little progress. In most cases where the DNA was intended for use in the solid state, for instance, in a film, the DNA was conjugated with other chemical compounds such as cationic lipids and polymers. ${ }^{2-5}$ Thus, fundamental knowledge about aggregation states and physical properties of DNA in a solid state has not been understood because the precise characterization of the DNA in the complex system is difficult. Previously, we have prepared self-supporting films of DNA by a low-environmental burden technique, that is, a solvent-casting method from its aqueous solutions, without the use of other chemicals. ${ }^{6}$ The thermal molecular motion of DNA chains in the film containing a given amount of water was studied by the dynamic mechanical analysis in conjunction with thermal analyses. The DNA in the film showed three relaxation processes that were markedly influenced by the presence of water in the film. That is, the relaxation temperatures decreased with an increasing water content. ${ }^{6}$ These results motivated us to make further studies on physical properties of DNA films, especially, in relation to the presence of water molecules. Here, we demonstrate distinctive tensile properties of the DNA film, which are lacking in other polymeric materials, and also, discuss its structural features in conjunction with the water content. Although a relatively dry DNA film behaves like a glassy polymer, its tensile property changes to a rubbery-like via a semicrystalline-like state solely by increasing the water content. To the best of our knowledge, this is the first report dealing with the 'glass'-'semicrystalline''rubber' transition for one material at a given temperature.

\section{EXPERIMENTAL PROCEDURES}

Commercially available powdered DNA sodium salt from salmon sperm (lot number EMW1909) with a broad molecular weight, ranging from $300 \mathrm{k}$ to $9 \mathrm{M}$, was purchased from Wako (Osaka, Japan) and used without further purification. The water content of the powdered DNA was estimated to be $12.2 \mathrm{wt} \%$ by thermogravimetric analysis using a SSC5200 (SII Nano Technology, Tokyo, Japan), and was comparable with a reported value by Lee et al. ${ }^{7}$ Thermogravimetric measurements were carried out at temperatures ranging from 303 to $473 \mathrm{~K}$ with a heating rate of $10 \mathrm{~K} \mathrm{~min}^{-1}$. DNA was dissolved into

${ }^{1}$ Department of Applied Chemistry, Kyushu University, Fukuoka, Japan; ${ }^{2}$ Japan Synchrotron Radiation Research Institute (JASRI), Sayo-gun, Japan and ${ }^{3}$ International Institute for Carbon-Neutral Energy Research (WPI-I2CNER), Kyushu University, Fukuoka, Japan

Correspondence: Dr H Matsuno or Professor K Tanaka, Department of Applied Chemistry, Kyushu University, 744 Motooka, Nishi-ku, Fukuoka 819-0395, Japan.

E-mail: h-matsuno@cstf.kyushu-u.ac.jp or k-tanaka@cstf.kyushu-u.ac.jp

Received 20 October 2013; revised 27 November 2013; accepted 15 December 2013 
ultrapure water purified by a Milli-Q system (Millipore, Billerica, MA, USA) Then, a concentrated aqueous DNA solution was cast onto a polystyrene dish. After allowing the water to evaporate by heating it for several days at $313 \mathrm{~K}$ and by successive vacuum drying at room temperature for $4 \mathrm{~h}$, a self-supporting transparent film with a thickness of $100 \mu \mathrm{m}$ was obtained. The films were then left under specified humidity conditions for several hours so that the water content in the films was controlled.

The mechanical properties of the DNA films were recorded on a standard tensile testing machine TENSILON RTC1250 (A\&D, Tokyo, Japan). The tensile tests were carried out at room temperature with a crosshead speed of $5 \mathrm{~mm} \mathrm{~min}^{-1}$. Fourier-transform infrared (FT-IR) spectra for the DNA films were recorded by a FT-IR620 (JASCO, Tokyo, Japan) spectrometer equipped with an MCT detector under ambient atmosphere at the resolution of $2 \mathrm{~cm}^{-1}$. The number of integrated spectra was 64. Wide-angle X-ray diffraction (WAXD) experiments were performed at the BL03XU beamline of SPring-8 (Japan Synchrotron Radiation Research Institute, Hyogo, Japan). ${ }^{8}$ The X-ray wavelength was $0.1 \mathrm{~nm}$ and the camera distance for the WAXD apparatus was $269 \mathrm{~mm}$. An imaging plate was used to record two-dimensional WAXD patterns. The calibration of the scattering angle was carried out using diffraction from a regular structure in silver behenate.

\section{RESULTS AND DISCUSSION}

Figure 1 shows the appearance of the self-supporting transparent DNA film. The water content was controlled by leaving a film in a humid environment.

Figure 2 shows the stress-strain (S-S) curves for DNA films with different water contents. The S-S curve for the $14-w t \%$ water film had a typical shape for a glassy polymer. We have previously confirmed that DNA chains in a film with a comparable water content retain a double-stranded helical structure at room temperature. ${ }^{6}$ In this case, double-stranded DNA (dsDNA) is a semiflexible polymer. ${ }^{9,10}$ The stress simply increased with increasing strain, as shown by curve (a). The Young's modulus determined from the initial slope of the $\mathrm{S}-\mathrm{S}$ curve was $\sim 1.7 \mathrm{GPa}$, which is a typical value for glassy polymers.

The glassy-like S-S behavior disappeared with an increasing water content in the film. In the case of a water content of $25 \mathrm{wt} \%$, amazingly, the S-S relationship was that of a semicrystalline polymer, as shown by curve (b). The curve could be divided into three regions; an initial elastic region, a necking zone and final strain-hardening region. In addition, the Young's modulus of the film decreased to $0.34 \mathrm{GPa}$. This change in the tensile properties of the film makes it clear that the aggregation states of dsDNA were altered by the invasion

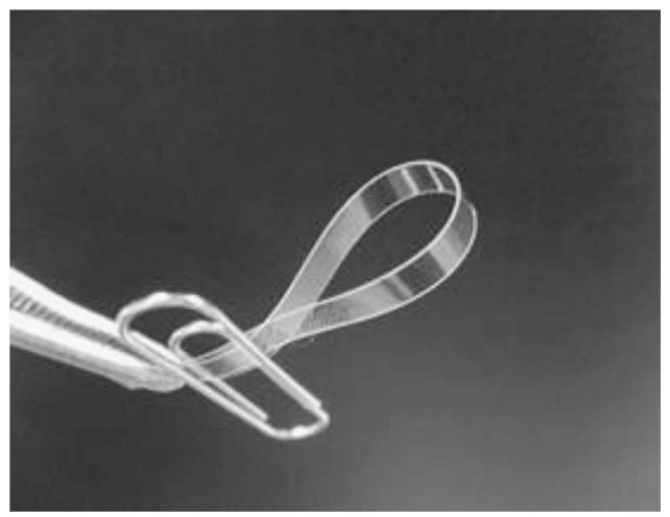

Figure 1 A self-supporting DNA film. The film was prepared by a simple solvent-casting method using commercially available sodium DNA powder extracted and purified from salmon testes. The water content of the film was $10 \mathrm{wt} \%$. of water molecules. Relatively short DNA with $150 \mathrm{bp}$ at a high concentration forms various liquid crystal phases in water depending on its concentration. ${ }^{11-13}$ The DNA used here is composed of 450-14000 bp, being much longer than that used in this previous literature. Hence, it seems reasonable to infer that dsDNA chains are partially oriented in the film, resulting in semicrystalline polymer-like tensile properties. Similar tensile properties could be observed for a DNA film with an even higher water content. However, the necking phenomenon became less marked with increasing water content. This is observed for the film with a water content of $31 \mathrm{wt} \%$, as depicted by curve (c). The Young's modulus for the film, which behaves a semicrystalline polymer in terms of the tensile properties, decreased with the increasing water content. These results make it clear that water molecules act as an effective plasticizer for DNA. As the water content was further increased to $46 \mathrm{wt} \%$, the film could be elongated up to a nominal strain of 6.05. The inset of Figure 2 shows an enlarged S-S curve for the 46-wt\% water film. Its Young's modulus has decreased to $7.5 \mathrm{MPa}$, which is a typical value for a rubbery polymer. The shape of this curve also looks like that of a rubbery polymer. In the case of a rubbery material, the elasticity manifested in the first and last sections of the S-S curve is typically explained in terms of entropy and energy elasticity, respectively. If the rubber is not chemically cross-linked, the latter part, namely stress hardening, will not be observed. Although the DNA used here was, of course, not cross-linked, stress hardening was still observed prior to the break. This behavior is quite unique.

As discussed above, the tensile properties of DNA could be changed from glassy to rubbery via semicrystalline-like at room temperature by simply regulating the water content in the film. To discuss why and how such unique properties can be realized, FT-IR spectroscopy and WAXD measurements were made. Figure 3A shows FT-IR spectra for the DNA films with water contents of (a) 12, (b) 47 and (c) 44 wt $\%$. The spectrum (c) was obtained under stretching at the nominal strain of 1.5. The sample for the spectrum (a) corresponds to the $14-\mathrm{wt} \%$ water film exhibiting the glassy-like S-S curve shown in Figure 2. Also, the samples for the spectra (b) and (c) correspond to the rubbery-like film with the water content of $46 \mathrm{wt} \%$ shown in Figure 2. Taking into account that the S-S curve for the $40-\mathrm{wt} \%$ water film (not shown) was almost identical to that for the $46 \mathrm{wt} \%$ one, it seems to reasonable to assume that there is no structural difference between 44 and $47 \mathrm{wt} \%$ films. Figure $3 \mathrm{~B}$ shows the enlarged spectra in the

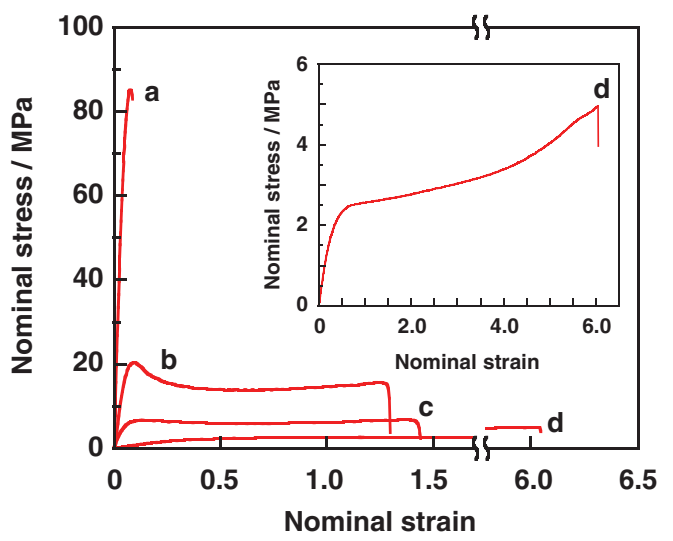

Figure 2 Stress-strain curves of the DNA films. Water content of the samples was (a) $14 w t \%$, (b) $25 w t \%$, (c) $31 w t \%$ and (d) $46 w t \%$, respectively. The measurements were performed at room temperature with a crosshead speed of $5 \mathrm{~mm} \mathrm{~min}^{-1}$. (Inset) Continuous plot for case (d). 

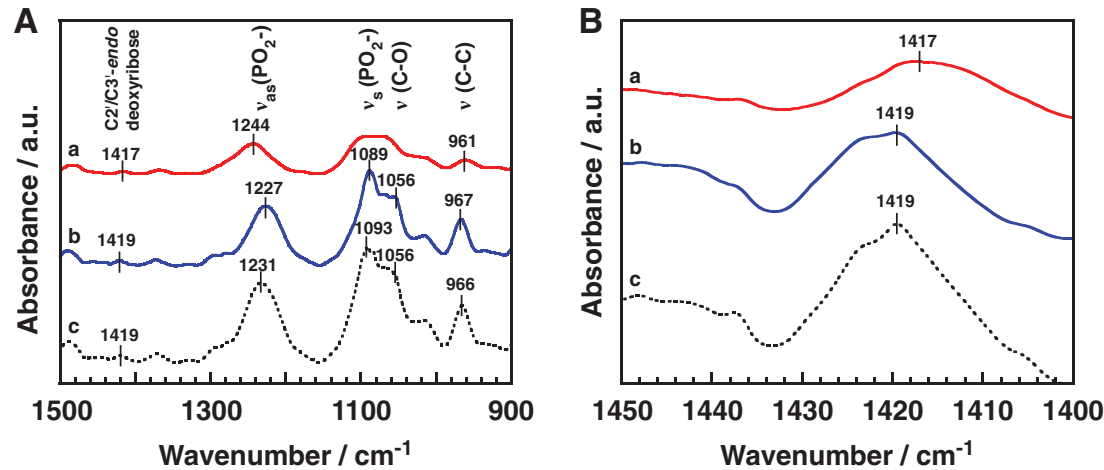

Figure 3 Fourier-transform infrared spectra of the DNA films. (A) The water content of each film was approximately (a, red) $12 \mathrm{wt} \%$, (b, blue) $47 \mathrm{wt} \%$ and (c, black) $44 \mathrm{wt} \%$, respectively. The unstretched films are shown as solid lines and the stretched film is shown as a dotted line. In the case of the stretched film, the strain was set to be 1.5. The five markers which signal the double-stranded DNA conformation, derived from the antisymmetric and symmetric stretching vibrations of phosphate $\left(v_{\mathrm{as}}\left(\mathrm{PO}_{2}^{-}\right)\right.$and $\left.v_{\mathrm{s}}\left(\mathrm{PO}_{2}^{-}\right)\right)$, the $\mathrm{C}-\mathrm{O}$ and $\mathrm{C}-\mathrm{C}$ stretch vibrations of backbone $(v(\mathrm{C}-0)$ and $v(\mathrm{C}-\mathrm{C}))$ and $\mathrm{C} 2^{\prime}-$ endo or $\mathrm{C}^{\prime}$-endodeoxyribose, were observed. (B) An expanded plot of the region from 1400 to $1450 \mathrm{~cm}^{-1}$. a.u., arbitrary unit.
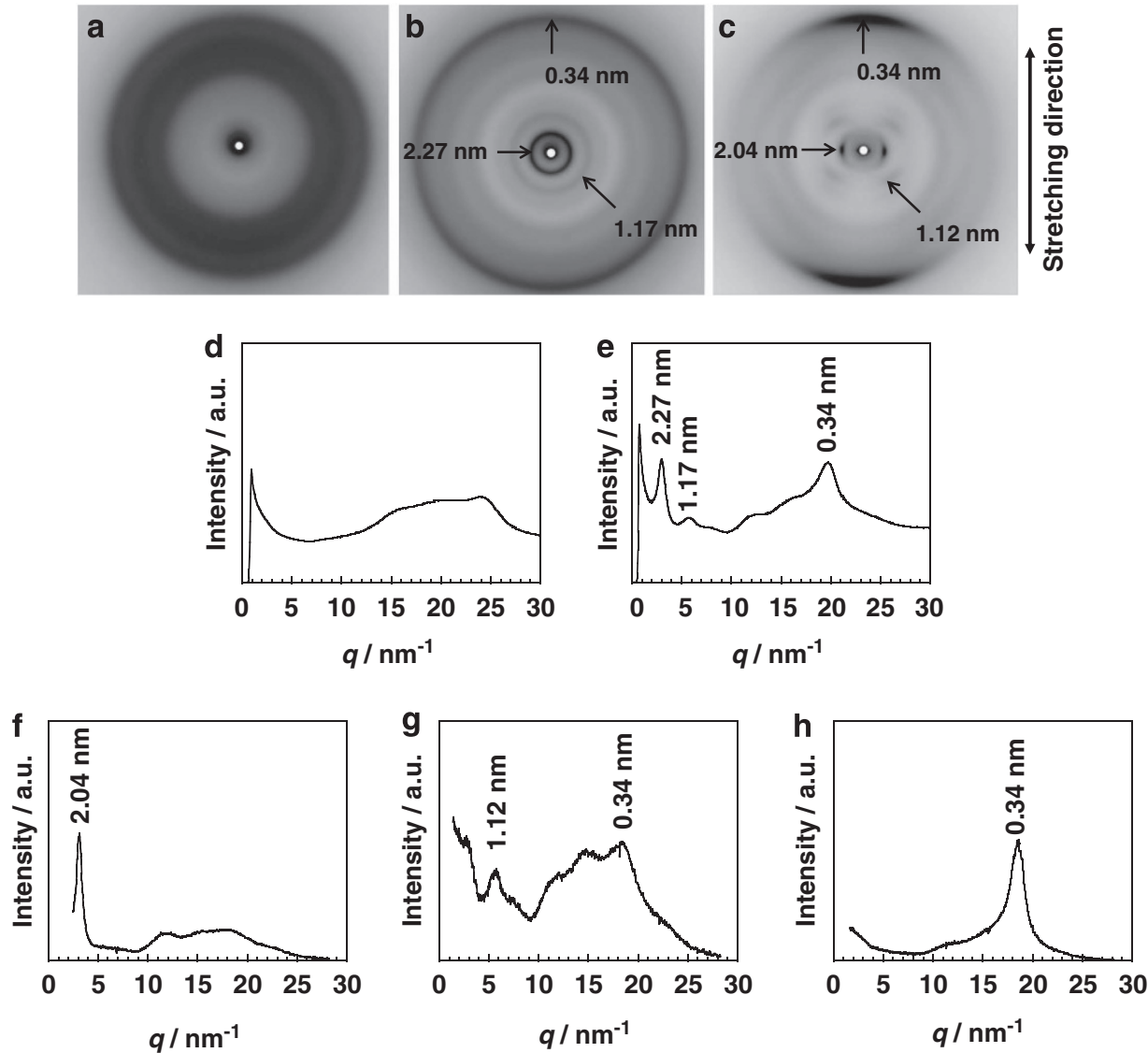

Figure 4 Two-dimensional patterns and one-dimensional profiles obtained by wide-angle X-ray diffraction analyses for the DNA films. Two-dimensional patterns of (a and b) unstretched and (c) stretched DNA films with water contents of (a) $12 \mathrm{wt} \%$, (b) $47 \mathrm{wt} \%$, and (c) $44 \mathrm{wt} \%$, respectively. The strain was set to be 1.5. (d and e) One-dimensional profiles obtained by integration of the full range of angles of pattern in panels a and $\mathbf{b}$, respectively. (f-h) One-dimensional profiles obtained by integration of small angle portion of the data ( $1^{\circ}$ ) of pattern (c) for (f) equatorial, (g) $45^{\circ}$ oblique and (h) vertical directions, respectively.

wavenumber range from 1450 to $1400 \mathrm{~cm}^{-1}$. Although dsDNA generally has a conformation of A- or B-form in nature, the B-form is dominant in living organisms. ${ }^{1}$ We here focus on the shift of five typical IR absorption bands, which signal the conformation of the dsDNA. In the case of the unstretched $12-\mathrm{wt} \%$ water film, the $\mathrm{C}-\mathrm{H}$ deformation vibration arising from the $\mathrm{C} 3^{\prime}$-endo deoxyribose was observed at $1417 \mathrm{~cm}^{-1}$, indicating that the dsDNA in the film adopted the A-form conformation. The peak shifted to $1419 \mathrm{~cm}^{-1}$ with increasing water content up to $\sim 47 \mathrm{wt} \%$, as shown in Figure $3 \mathrm{~B}$. This shift reflects a conformational change of the deoxyribose portion from $\mathrm{C}^{\prime}$-endo to $\mathrm{C} 2^{\prime}$-endo, that is, a conformational change from the A- to the B-form of dsDNA in the film. ${ }^{14,15}$ This result is in good 
accord with our knowledge that the A-form is usually formed under dehydrated conditions. ${ }^{1}$

The wavenumber of the antisymmetric and symmetric stretching vibrations of $\mathrm{PO}_{2}^{-}\left(v_{\text {as }}\left(\mathrm{PO}_{2}^{-}\right)\right.$and $\left.v_{s}\left(\mathrm{PO}_{2}^{-}\right)\right)$, which are also typical markers for the conformation of dsDNA backbone, independent of nucleobase vibrations and sugar pucker, ${ }^{14,15}$ changed with increasing water content. Usually, $v_{\mathrm{as}}\left(\mathrm{PO}_{2}{ }^{-}\right)$for the $\mathrm{A}$ - and $\mathrm{B}$-forms appears around $1240 \mathrm{~cm}^{-1}$ and $1225 \mathrm{~cm}^{-1}$, respectively. ${ }^{14,15}$ Although the peak was observed at $1244 \mathrm{~cm}^{-1}$ for the film with the water content of $12 \mathrm{wt} \%$, it shifted to $1227 \mathrm{~cm}^{-1}$ for the film with the water content of $47 \mathrm{wt} \%$. Also, the peak attributed to $v_{\mathrm{s}}\left(\mathrm{PO}_{2}{ }^{-}\right)$around approximately $1089 \mathrm{~cm}^{-1}$ became apparent with increasing water content. In addition, a typical marker for the B-form arising from the stretching vibration of $\mathrm{C}-\mathrm{O}$ bonds on the deoxyribose $(v(\mathrm{C}-\mathrm{O}))$ was observed at $1056 \mathrm{~cm}^{-1}$ only for the films with higher water content. An absorption peak assignable to the $\mathrm{C}-\mathrm{C}$ stretching vibration of deoxyribose $(v(\mathrm{C}-\mathrm{C}))$ was observed at 961 and $967 \mathrm{~cm}^{-1}$ for the films with the lower and higher water contents, respectively. The former and latter peak positions are the signature of the A- and B-forms, respectively. ${ }^{14}$ These results also make it clear that the conformation of dsDNA changed from A- to B-form with increasing the water content in the film. ${ }^{14,15}$

The spectrum of the film with a similar water content of $44 \mathrm{wt} \%$, but stretched with a strain of 1.5 , is also shown in Figure 3 . It is almost the same as the unstretched one except for the $v_{\mathrm{as}}\left(\mathrm{PO}_{2}{ }^{-}\right)$ peak, which is shifted from 1227 to $1231 \mathrm{~cm}^{-1}$. Unfortunately, we have no obvious explanation for this shift at the moment. However, considering that neither shifts nor intensity changes for other characteristic markers were observed, it is conceivable that the conformation of dsDNA in the film remained in the B-form even after stretching. This will be discussed in the context of the WAXD data later.
The aggregation states of DNA in the films were also examined by WAXD. Figure 4 shows the examples of typical WAXD patterns obtained. The films for the patterns in panels $(a-c)$ correspond to the ones exhibiting the respective $\mathrm{S}-\mathrm{S}$ curves for typical glassy and rubbery polymers. Hence, the patterns in panels $(\mathrm{a}-\mathrm{c})$ can be directly compared with the FT-IR spectra $(\mathrm{a}-\mathrm{c})$ shown in Figure 3. In the case of the water content of $12 \mathrm{wt} \%$, an amorphous halo was observed without clear periodic rings. Taking into account the FT-IR result, it is clear that dsDNA in the film with lower water content takes the A-form conformation, being in an amorphous state. This is consistent with its tensile properties, which correspond to those of a glassy polymer.

On increasing the water content to $47 \mathrm{wt} \%$, three diffraction rings corresponding to the distances of $0.34,1.17$ and $2.27 \mathrm{~nm}$ were clearly observed. The distance between one pair of bases in the B-form of dsDNA has been reported to be $0.34 \mathrm{~nm}$, which is exactly the same as the length of $0.34 \mathrm{~nm}$ observed here. ${ }^{1}$ That is, the WAXD measurement shows that the A-form conformation of dsDNA is transformed to the B-form with increasing water content in the solid film. The distance of $2.27 \mathrm{~nm}$ is slightly larger than the reported diameter of the typical B-form DNA, $2.0 \mathrm{~nm} .{ }^{1}$ Thus, it seems reasonable to infer that the $2.27-\mathrm{nm}$ distance represents the distance between dsDNA chains. If that is the case, the dsDNA helix should be loosely packed and such a structure then leads to a rubbery behavior upon stretching.

After stretching the film, the WAXD pattern was drastically changed from the original, as shown in Figure 4c. Diffraction arcs corresponding to a distance of $0.34 \mathrm{~nm}$ were observed along the meridian direction namely, parallel to the stretching direction. Also, other diffraction arcs were observed along the equatorial direction and correspond to a distance of $2.04 \mathrm{~nm}$. The former and latter values are almost equal to the distances between base pairs and
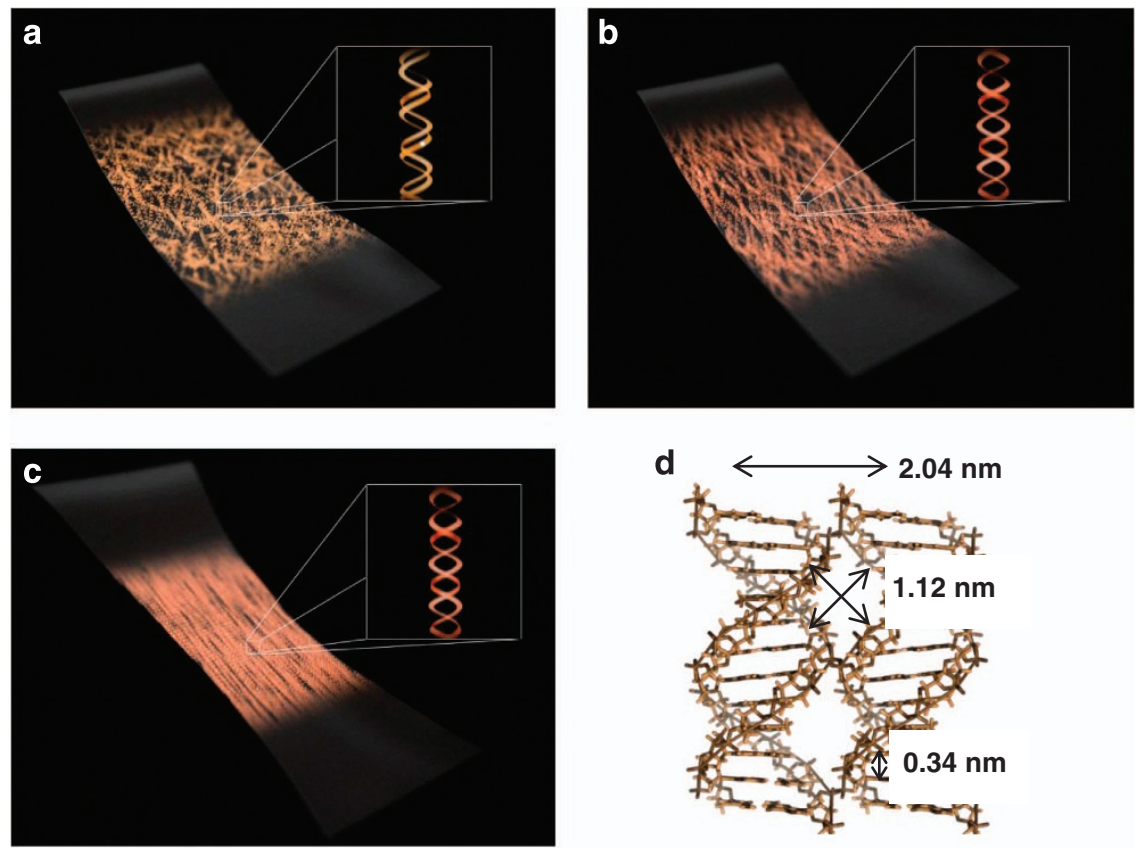

Figure 5 Schematic illustrations of the DNA films. (a) The unstretched film with low water content. The conformation is A-form and the aggregation state is amorphous. (b) The unstretched film with high water content. The conformation is B-form and chains are partially packed. (c) The stretched film with high water content. The conformation is also B-form. (d) Schematic model of the aggregation state of stretched B-form double-stranded DNA. The distances obtained by wide-angle X-ray diffraction analyses are shown. 
the diameter of the B-form dsDNA, respectively; meaning that dsDNA chains are highly oriented along the stretching direction. Furthermore, a butterfly pattern that is thought to originate from a distance of $1.12 \mathrm{~nm}$ was observed. This value could be explained in terms of the distance between two adjacent dsDNA backbones.

In conclusion, we found peculiar tensile properties of solid films of DNA, which is a perfect green material. The tensile response of a DNA film could be changed from glassy to rubbery via semicrystalline-like by simply regulating the water content in the film. The state transition, which has never been found in any kind of synthetic polymers, can be associated with changes in the aggregation states of dsDNA induced by the water content, as shown in Figure 5 . In a relatively dry state, the DNA is in the A-form conformation. It transforms from the A-form to the B-form with increasing water content. Even if the film is stretched, conformation of DNA remains in the B-form, but becomes highly oriented parallel to the stretching direction. We believe that structural synthetic polymers may be replaced with this perfect green polymer in the near future and that the information obtained here will be useful for the design of novel functional materials using natural polymers instead of synthetic polymers.

\section{CONFLICT OF INTEREST}

The authors declare no conflict of interest.

\section{ACKNOWLEDGEMENTS}

We thank Dr Yoshihisa Fujii and Dr Tomoyasu Hirai in Kyushu University, and Mr Junichiro Koike and Dr Misao Horigome in DIC Corporation for fruitful discussions, especially concerning the WAXD analysis. This research was partly supported by the Scientific Research on Innovative Areas 'Molecular Soft-Interface Science' (no. 23106716) and 'New Polymeric Materials Based on Element-Blocks' (no. 25102535) programs and by a Grant-in-Aid for Scientific Research (B) (no. 24350061) from the Ministry of Education, Culture, Sports, Science and Technology, Japan. The WAXD measurements were carried out at BL03XU at SPring-8 with the proposal number 2011B 7279, 2011B 7280 and 2012A 7228
1 Saenger, W. Principles of Nucleic Acid Structure (Springer-Verlag, New York, NY, USA, 1987).

2 Okahata, Y., Kobayashi, T. \& Tanaka, K. Orientation of DNA double strands in a Langmuir-Blodgett film. Langmuir 12, 1326-1330 (1996).

3 Wang, L., Yoshida, J., Ogata, N., Sasaki, S. \& Kajiyama, T. Self-assembled supramolecular films derived from marine deoxyribonucleic acid (DNA)-cationic surfactant complexes: large-scale preparation and optical and thermal properties. Chem. Mater. 13, 1273-1281 (2001).

4 Nagamura, T., Yamamoto, M., Terasawa, M. \& Shiratori, K. High performance sensing of nitrogen oxides by surface plasmon resonance excited fluorescence of dye-doped deoxyribonucleic acid thin films. Appl. Phys. Lett. 83, 803-805 (2003).

5 Smitthipong, W., Neumann, T., Gajria, S., Li, Y., Chworos, A., Jaeger, L. \& Tirrell, M. Noncovalent self-assembling nucleic acid-lipid based materials. Biomacromolecules 10, 221-228 (2009)

6 Matsuno, H., Nakahara, J. \& Tanaka, K. Dynamic mechanical properties of solid films of deoxyribonucleic acid. Biomacromolecules 12, 173-178 (2011).

7 Lee, S., Debenedetti, P., Errington, J., Pethica, B. \& Moore, D. A Calorimetric and spectroscopic study of DNA at low hydration. J. Phys. Chem. B 108, 3098-3106 (2004).

8 Masunaga, H., Ogawa, H., Takano, T., Sasaki, S., Goto, S., Tanaka, T., Seike, T., Takahashi, S., Takeshita, K., Nariyama, N., Ohashi, H., Ohata, T., Furukawa, Y., Matsushita, T., Ishizawa, Y., Yagi, N., Takata, M., Kitamura, H., Sakurai, K., Tashiro, K., Takahara, A., Amamiya, Y., Horie, K., Takenaka, M., Kanaya, T., Jinnai, H., Okuda, H., Akiba, I., Takahashi, I., Yamamoto, K., Hikosaka, M., Sakurai, S., Shinohara, Y., Okada, A. \& Sugihara, Y. Multipurpose soft-material SAXS/WAXS/GISAXS beamline at SPring-8. Polymer J. 43, 471-477 (2011)

9 Cifra, P., Benková, Z. \& Bleha, T. Persistence length of DNA molecules confined in nanochannels. Phys. Chem. Chem. Phys. 12, 8934-8942 (2010).

10 Gutjahr, P., Lipowsky, R. \& Kierfeld, J. Persistence length of semiflexible polymers and bending rigidity renormalization. Europhys. Lett. 76, 994-1000 (2006).

11 Pelta, Jr J., Durand, D., Doucet, J. \& Livolant, F. DNA mesophases induced by spermidine: structural properties and biological implications. Biophys. J. 71, 48-63 (1996).

12 Livolant, F., Levelut, M., Doucet, J. \& Benoit, P. The highly concentrated liquidcrystalline phase of DNA is columnar hexagonal. Nature 339, 724-726 (1989).

13 Strzelecka, E., Davidson, W. \& Rill, L. Multiple liquid crystal phases of DNA at high concentrations. Nature 331, 457-460 (1988).

14 Whelan, D. R., Bambery, K. R., Heraud, P., Tobin, M. J., Diem, M., McNaughton, D. \& Wood, B. R. Monitoring the reversible B to A-like transition of DNA in eukaryotic cells using Fourier transform infrared spectroscopy. Nucleic Acids Res. 39, 5439-5448 (2011).

15 Banyay, M., Sarkar, M. \& Graslund, A. A library of IR bands of nucleic acids in solution. Biophys. Chem. 104, 477-488 (2003).

(c) (1)(2) This work is licensed under a Creative Commons Attribution-NonCommercial-ShareAlike 3.0 Unported License. To view a copy of this license, visit http://creativecommons. org/licenses/by-nc-sa/3.0/ 